\title{
The observed change in the value of 'fine structure constant' can be interpreted through the consciousness model
}

\author{
Dhananjay Pal \\ Email address: \\ dhananjay.pal123@gmail.com,paldhananjay46@yahoo.com
}

Pharmacy College, Bengal School of Technology, Sugandha-Delhi Road, Chuchura, Dist.-Hooghly, West Bengal, INDIA

\section{To cite this article:}

Dhananjay Pal. The Observed Change in the Value of 'Fine Structure Constant' can be Interpreted through the Consciousness Model. American Journal of Modern Physics. Vol. 3, No. 1, 2014, pp. 8-15. doi: 10.11648/j.ajmp.20140301.12

\begin{abstract}
A single field emerged at the origin of the universe, already containing within itself the blueprint of the physical universe. The primordial single field triggered the onset of the universe. Most physicists believe that a single super-force dominated the first instants of creation. Scientists have arrived at a simple but decisive conclusion that consciousness is very much a part of the universe, like other objects. Our consciousness model involving thought-carrying particle (TCP), thought retaining particle (TRP) and thought force $\left(\mathrm{T}_{\mathrm{F}}\right)$ signifies the existence of universal consciousness that exists along with the universe. This universal consciousness is a functional state of the universal mind (UM). This UM is evolved at the Big Bang from void. The UM is constituted by these TCP and TRP in the inherent presence of thought force $\left(\mathrm{T}_{\mathrm{F}}\right)$. Thought force $\left(\mathrm{T}_{\mathrm{F}}\right)$ is an expression of universal consciousness. The Thought force $\left(\mathrm{T}_{\mathrm{F}}\right)$ being the primordial quantum field functions as the original super-force. $\mathrm{T}_{\mathrm{F}}$ being the original super-force functions as the origin of all the fundamental fields. TCP is the carrier of thought force $\left(\mathrm{T}_{\mathrm{F}}\right)$ that, in turn, appears to be the origin of all the fields. The quantized energy $\left(\varepsilon_{T}\right)$ of TCP is responsible to cause the universal consciousness as well as the cosmic microwave background radiation temperature. The individual consciousness owes its origin to the universal consciousness created by the same $\mathcal{E}_{T}$. The same $\varepsilon_{T}$ is the energy responsible for generating thought force $\left(\mathrm{T}_{\mathrm{F}}\right)$. $\mathrm{T}_{\mathrm{F}}$ being an expression of the universal consciousness is applicable to any inanimate object as well as to any biological system (having thinking ability). The $\mathrm{T}_{\mathrm{F}}$ exerts its functions both in vitro and in vivo. The consciousness model involving the quantum mechanical activities of these TCP, TRP and thought force may provide guidelines to interpret the observed change in the value of 'fine structure constant'.
\end{abstract}

Keywords: Void, Universal mind (UM), Thought Force $\left(\mathrm{T}_{\mathrm{F}}\right)$, Though-Carrying Particles (TCP), Thought Retaining Particles (TRP), Quantized Energy $\left(\in_{\mathrm{T}}\right)$ of TCP, Cosmic Microwave Background Radiation (CMBR), Universe Wide Web (uww)

\section{Introduction}

In Eastern philosophical traditions, consciousness is intrinsic to the universe, whereas in most Western views, consciousness is extrinsic, emerging from complex computation. How can these views be reconciled?

It is most relevant and important to indicate the names of various eminent physicists like Erwin Schrödinger, Eugene Wigner, Brian Josephson, John Wheeler, Roger Penrose, Henry P Stapp, Freeman J. Dyson, Paul Davies, David Bohm, Basil Hiley, Fritjof Capra, Fred Alan Wolf and Amit Goswami who have addressed the inclusion of consciousness in their work. Consciousness is to be taken into account.
1.1. In contrast to the usual linear sequence of matter, body, life, brain, mind, consciousness, here the proposed cyclic sequence is first universal consciousness (a functional state of the universal mind), and then matter, body, life, brain, and regeneration of mind and consciousness. The evolution of life with mind and consciousness is possible purely due to the inherent existence of universal consciousness which exists along with the universe. The human nervous system is evolved to provide an appropriate material structure to individualize the universal consciousness, a characteristic of reality, 
pervading all manifestations.

Consciousness model of Pal et al [1-3] involving TCP, TRP and thought force $\left(\mathrm{T}_{\mathrm{F}}\right)$ signifies the existence of universal consciousness that exists along with the universe. Pal et al [3] showed that this universal consciousness is a functional state of Universal Mind (UM). Pal et al [3] explained that the UM is evolved at the Big Bang from the eternal Void. This Void, in turn, is the source of infinite energy. And this $\mathrm{UM}$ is a finer matter. The individual mind being a constituent of the UM is also a finer matter. The constituents of the UM and individual mind are the same. The ultimate constituents of matter and mind are the same as both mind and matter are aspects of one fundamental reality, which is called UM. The brain is the mediating link or interface between the individual mind and body.

Pal et al [3] explained that the constituents of the UM are the ultimate constituents of matter itself as everything in this universe is a manifestation of this UM. Pal et al [3] expressed that the UM is constituted by these TCP and TRP in the inherent presence of thought force $\left(\mathrm{T}_{\mathrm{F}}\right)$. Pal et al $[1,3]$ further explained that the ultimate constituents of matter and mind are these TCP and TRP in the inherent presence of thought force $\left(\mathrm{T}_{\mathrm{F}}\right)$ in vitro and thought force $\left(\mathrm{T}_{\mathrm{F}}\right)$ in vivo.

Physicists determined that underlying quantum fields give birth to elementary particles. Bhaumik [4] mentioned that Frank Wilczek pointed out, "In quantum field theory, the primary elements of reality are not individual particles, but underlying fields. Thus, for example, all electrons are but excitations of an underlying field, naturally called electric field". The same holds true for all the fundamental particles of which matter is made.

The existence of matter depends on the existence of force and vice versa. TCP cannot exist without TRP and vice versa. Many physicists believe that unifying all the forces, including gravity, into a single theory would require a phenomenon called super-symmetry. With supersymmetry, every fermion would have a boson twin, and vice-versa. The thought force $\left(\mathrm{T}_{\mathrm{F}}\right)$ is carried by the TCP in the presence of its super-symmetrical partner TRP. TCP that behaves like boson should accompany its supersymmetrical partner TRP that functions like fermion in the generalized simpler way. It is to be noted that these TCP and TRP function like wavicle: wave-particle duality.

\section{Thought Force}

Pal et al [1,3] and Pal [5] explained the existence of thought force (TF). Thought force (TF), an expression of the universal consciousness, is the primordial quantum field that, in turn, functions as the primary unified field. This TF being an expression of the universal consciousness is applicable to any inanimate object as well as to any biological system (having thinking ability). Thus the TF being an expression of the universal consciousness exerts its functions both in vitro and in vivo.

Physicists determined that underlying quantum fields give birth to elementary particles. Pal [5] expressed that the thought force $\left(\mathrm{T}_{\mathrm{F}}\right)$ is the primordial quantum field. Thought force $\left(T_{F}\right)$ being the primordial quantum field functions as the primary unified field. Thought force $\left(\mathrm{T}_{\mathrm{F}}\right)$ being the primordial quantum field gives birth to TRP that appears to be the origin of all the matter particles. TCP is the carrier of thought force $\left(\mathrm{T}_{\mathrm{F}}\right)$ that, in turn, appears to be the origin of all the fields. TCP thus appears to be the origin of all the field particles.

In a purpose to involve both the non-living and living systems of the world, Pal [5] has shown the existences of these TCP, TRP and thought force $\left(\mathrm{T}_{\mathrm{F}}\right)$ in vitro and thought force $\left(\mathrm{T}_{\mathrm{F}}\right)$ in vivo. Anyone can call this TCP by any other name, but as the highly developed living system will have to be evolved in the universe in the long run and as the thought of highly developed living system appears to be a kind of force to be called the thought force $\left(\mathrm{T}_{\mathrm{F}}\right)$ in vivo, we considered it is wise to call it as TCP. Further, as the universe exists along with the universal consciousness that, in turn, is created by the quantized energy $\left(\varepsilon_{T}\right)$ of TCP, we had to use the term TCP.

Pal (5) expressed that the non-living system of the world is governed by the thought force (TF) in vitro and this Thought force (TF) in vitro gives rise to TF (micro), SNF, EMF, WNF, GF and TF (macro) where TF (micro) = Thought force in microcosm, SNF = Strong nuclear force, $\mathrm{EMF}=$ Electromagnetic force, $\mathrm{WNF}=$ Weak nuclear force, $\mathrm{GF}=$ Gravitational force and TF (macro) $=$ Thought force in macrocosm. It is to be noted here that TF (micro) is a stronger force than the SNF and TF (macro) is a weaker force even than the GF.

Pal (5) also expressed that the living system of the world is governed by the thought force (TF) in vivo and this Thought force (TF) in vivo is a type of force that represents the biological 'thought' which is the action of mind. This 'thought' being a type of force controls the 'thought processes' involving the firing of neurons through the quantum mechanical activities of these TCP and TRP in the presence of consciousness. Consciousness in living organisms is a process which involves the quantum mechanical activities of these TCP and TRP, the ultimate constituents of any matter as well as any mind in the inherent presence of thought force (TF) in vitro and the thought force (TF) in vivo as indicated by Pal et al [3]. This consciousness, in turn, is the quantized energy $\left(\varepsilon_{T}\right)$ of TCP. The thought force (TF) in vivo is demonstrated in numerous experiments in which thought has an effect on a physical process (often known as mind over matter). This biological 'thought' is a type of force that can cause movement. Controlling movement through thought alone is observed in several experiments conducted by many scientists as indicated by Pal (5). These experiments thus signify the existence of thought force (TF) in vivo.

Pal [5] explained the existence of $\mathrm{T}_{\mathrm{F}}$ (micro) $(=$ Thought force in microcosm). This $\mathrm{T}_{\mathrm{F}}$ (micro) is the strongest interaction (a new class of 'extra strong' interaction). It is stronger than SNF (Strong Nuclear Force).

Pal [5] has also shown the existence of $\mathrm{T}_{\mathrm{F}}$ (macro) $(=$ 
Thought force in macrocosm). It is the "weakest force" which is much weaker even than the gravity.

2.1. Pal et al [3] developed three different equations expressing the quantized energy ( $\left.\mathcal{E}_{T}\right)$ of TCP. The value of $\mathcal{E}_{T}$ in one of the three equations is shown below:

$$
\varepsilon_{T}=4.384 \times 10^{-16} \mathrm{erg} \equiv 2.73 \times 10^{-4} \mathrm{eV} \equiv 2.73 \mathrm{~K} \cong C M B R \text { temperature } \equiv 2.725 \mathrm{~K} .
$$

This conversion of $\mathrm{erg} \equiv \mathrm{eV} \equiv \mathrm{K}$ is given by Weisskopf [6] as follows:

$$
1 e r g \cong 0.6241807 \times 10^{12} \mathrm{eV}^{\text {and }} 10^{-4} \mathrm{eV} \cong 1 \mathrm{~K}
$$

2.2. Pal et al [3] expressed that the CMBR temperature is due to $\varepsilon_{T}$, the quantized energy of TCP where

$$
\varepsilon_{T}=4.384 \times 10^{-16} \mathrm{erg} \equiv 2.73 \times 10^{-4} \mathrm{eV} \equiv 2.73 \mathrm{~K} \cong C M B R \text { temperature } \equiv 2.725 \mathrm{~K} .
$$

As per Pal et al [3], it is interesting to note that all of the three different equations ultimately give rise to the same result that is equivalent to the CMBR temperature. A sort of relationship is thus observed between the $\varepsilon_{T}$ and the CMBR temperature. This coincidence is thus signifying a probable role of TCP on the maintenance of CMBR temperature. Further, this coincidence is also signifying the existence of these TCP in the presence of TRP. TCP cannot exist without TRP and vice versa. The presence of TCP, TRP and Thought force $\left(\mathrm{T}_{\mathrm{F}}\right)$ in the universe is thus indicated and expressed mathematically. At present we are unable to explain when and how these TCP and TRP decoupled from the primordial cosmic soup.

Pal et al $[1,3]$ showed that the quantized energy $\left(\varepsilon_{T}\right)$ of TCP is responsible to cause the universal consciousness as well as the cosmic microwave background radiation temperature. The individual consciousness owes its origin to the universal consciousness created by the same $\varepsilon_{T}$. Ultimately this $\varepsilon_{T}$ represents universal consciousness. The existence of CMBR temperature indicates the existence of the TCP in the presence of TRP. The existence of TCP ensures the existence of the thought force $\left(\mathrm{T}_{\mathrm{F}}\right)$. Further, this $\mathrm{T}_{\mathrm{F}}$ being an expression of the quantized energy $\left(\boldsymbol{\varepsilon}_{T}\right)$ of TCP exerts its functions both in vitro and in vivo.

\section{What is Life?}

Life is a state of flux that is being maintained by a typical form of energy which is nothing but the consciousness itself. Pal et al [3] and Pal [7] showed that the quantized energy $\left(\varepsilon_{T}\right)$ of TCP is responsible to cause the universal consciousness as well as the cosmic microwave background radiation temperature. The individual consciousness owes its origin to the universal consciousness created by the same $\varepsilon_{T}$. Pal et al $[1,3]$ and Pal [7] explained that life may be defined as a state of functional manifestation of consciousness that, in turn, is the quantized energy $\left(\mathcal{E}_{T}\right)$ of TCP. This $\mathcal{E}_{T}$ represents universal consciousness.

Thus,

$$
\text { Life }=\mathrm{f}(\text { Consciousness })=\mathrm{f}\left(\boldsymbol{\varepsilon}_{T}\right)
$$

where $\varepsilon_{T}=$ quantized energy of the TCP $=4.384 \times 10^{-16} \mathrm{erg}$.

$$
\varepsilon_{T}=h v_{T}=h c / \lambda_{T}=4.384 \times 10^{-16} \mathrm{erg}
$$

where

$$
\begin{aligned}
& \mathcal{E}_{T}=\text { quantized energy of the TCP }=4.384 \times 10^{-16} \mathrm{erg}, \\
& \boldsymbol{v}_{T}=\text { frequency of the TCP }=\varepsilon_{T} / \mathrm{h}=66.12 \times 10^{9} \mathrm{~Hz} \cdot=66.12 \mathrm{GHz}, \\
& \mathrm{h}=\text { Planck's quantum constant }=6.63 \times 10^{-27} \mathrm{erg} \cdot \mathrm{sec}, \\
& \mathrm{c}=\text { free-space velocity of light }=3 \times 10^{10} \mathrm{~cm} / \mathrm{sec}, \\
& \lambda_{T}=\text { wave-length of the } \mathrm{TCP}=0.4537 \mathrm{~cm} .
\end{aligned}
$$

\subsection{What is Consciousness?}

Psychologists, neuroscientists, philosophers, and other professionals continue to engage in an ongoing debate as to what consciousness means. In reality, we may never know. Is it a product of the biological and classical physical interactions of the human brain; or is it something more fundamental, perhaps electromagnetic, or the result of quantum physics principles that we don't yet fully understand? Could it be something even more profound than that -- something beyond the scope of science and physics, any kind of physics, for us to understand?

According to the ancient Vedanta, consciousness is not an emergent property of matter that comes into existence only through the functioning of the human nervous system. Instead, consciousness is a characteristic of reality, pervading all manifestations. This unbounded field of nature's universal consciousness is not limited to an individual consciousness. From this viewpoint, the role of the human nervous system is to provide an appropriate material structure to individualize the universal consciousness. It appears that inanimate matter itself cannot generate consciousness without the inherent existence of universal consciousness.

Pal et al [2] explained, "Consciousness is the realization of existence, and there are as many states of consciousness as there are states of existence. Every living being has a consciousness of its own depending on complexity of the brain and the activity of the viable numbers of TCP in the 
presence of TRP; and the state of its consciousness changes every moment of time. Consciousness is the perception of the relation it bears to things and as this relation changes, consciousness changes its character. Consciousness itself does not change; it only moves up and down on the 'scale of the realization' of existence through the 'sub-conscious', 'conscious' and 'super-conscious' states". There is another term called unconscious state. The most usual unconscious state is sleep. A deeper form of unconsciousness is called a coma.

\subsection{Possible Relation of Consciousness with Mind: Possible Relation of Animate with Inanimate Through Consciousness}

Modern scientists have not even been able to arrive at a consensus on what should be a definition of the totality of consciousness. The brain is intricately linked to the process of consciousness and consciousness is thought to be a phenomenon of the mind.

It appears that

$$
\text { Consciousness }=\mathrm{f}(\text { mind })
$$

Consciousness is the functional state of mind. Presence of consciousness signifies the presence of mind and vice versa.

It is apparent that

$$
\text { Animate } \neq \text { Inanimate }
$$

Three critical factors which distinguish life from nonliving are consciousness, metabolism and reproduction.

In a gross presentation, we can express from the equation (4):

$$
\text { Animate }- \text { Consciousness }=\text { Inanimate }
$$

Scientists would have to define and characterize consciousness properly. We should have the proper knowledge about the exact characteristics of consciousness in order to address many present day scientific enigmas.

\subsection{Consciousness may be Defined as the 'Self-Organized' Capability of any Living Being to Activate TCP and TRP}

Pal et al [3] expressed that consciousness may be defined as the 'self-organized' capability of any living being to activate TCP and TRP, the ultimate constituents of mind and matter and to exert its functions. What can generate, maintain and activate TCP and TRP is called animate having 'active consciousness' and what cannot is called inanimate, i.e., devoid of active consciousness. As anything inanimate does neither have the power to activate the TCP and TRP nor have the capability to catalyze the activity of TCP and TRP, so it cannot generate consciousness. On the contrary, anything animate has the 'self-organized' power to generate, activate and catalyze the activity of TCP and TRP in order to generate and maintain consciousness along with the vital living force. Prigogine et al [8] explained "Self-Organization in Non-Equilibrium Systems".

Consciousness in living organisms is a process which involves the quantum mechanical activities of these TCP and TRP, the ultimate constituents of any matter as well as any mind in the inherent presence of thought force $\left(\mathrm{T}_{\mathrm{F}}\right)$ in vitro and the thought force $\left(\mathrm{T}_{\mathrm{F}}\right)$ in vivo as indicated by Pal et al [1,3]. And these TCP and TRP govern the activities of neurons (not the other way round). Neurons are simply the equipments used to generate consciousness and awareness. The consciousness itself is functioning as an inter-linking agent between the animate and inanimate through the quantum mechanical activities of these TCP and TRP indicated by [3].

\section{Existence of Universal Consciousness}

Pal et al [3] and Pal [7] showed the existence of universal consciousness and explained that the quantized energy $\left(\varepsilon_{T}\right)$ of TCP is responsible to cause the universal consciousness as well as the cosmic microwave background radiation temperature. The individual consciousness owes its origin to the universal consciousness created by the same $\varepsilon_{T}$.

Pal et al [3] have expressed that according to the modern physicists, the universe is the summation of fields, particles, space-time continuum, dark matter, dark energy, void and all the known and unknown parameters of the universe along with all its inhabitants (with or without consciousness), although the universe is always expanding.

The method of integration indicates that

$$
\int d x=x+c
$$

where $\mathrm{c}=\mathrm{a}$ constant. Is there any mathematical fallacy if we like to use 'universe' in the place of ' $x$ ' here? If we at all theoretically like to apply the same method of integration to the whole universe itself, we will have to arrive at

$$
\int d(\text { universe })=\text { universe }+c
$$

where universe $=$ universe with all its known and unknown parameters as well as its inhabitants (with or without consciousness) and $\mathrm{c}=\mathrm{a}$ constant. The constituents of the universe are fields, particles, space-time continuum, dark matter, dark energy, void, all its inhabitants (with or without consciousness) and all the other unknown parameters of the universe that we have not yet faced. We simply propose to apply the method of integration theoretically over the universe as a whole with all its known and unknown parameters and all the inhabitants (with or without consciousness). If we at all theoretically like to do so, we will mathematically arrive at a constant.

What is this constant here? It is our opinion that this constant is the universal consciousness that exists throughout the universe in the form of universe wide web 
(uww) covering fields, particles, space-time continuum, dark matter, dark energy, void and all its known and unknown parameters along with all its inhabitants (with or without consciousness). This universal consciousness is to be taken into account, but usually ignored.

Many physicists agree with the idea that consciousness is nonlocal, fundamental in the universe and consciousness is very much a part of the universe, like other objects. It is to be noted that $\varepsilon_{T}$, the quantized energy of TCP represents universal consciousness.

Nelson [9], Director of Global Consciousness Project, has observed through the experimentation that coherent consciousness creates order in the world; and subtle interactions link us with each other and the Earth. When human consciousness becomes coherent and synchronized, the behavior of random systems may change. Quantum event based random number generators (RNGs) produce completely unpredictable sequences of zeroes and ones. But when a great event synchronizes the feelings of millions of people, our network of RNGs becomes subtly structured. The probability is less than one in a billion that the effect is due to chance. The evidence suggests an emerging noosphere, or the unifying field of consciousness described by sages in all cultures. This evidence signifies and proves the existence of universal consciousness.

$$
\varepsilon_{T}=\text { quantized energy of the TCP }=\left(\frac{h c}{\lambda_{T}}\right)=\left(\frac{h^{3} c^{5} m}{V_{p r}}\right)^{1 / 4}=4.95 \times 10^{-16} \mathrm{erg},
$$

where $\lambda_{T}=$ wavelength in $\mathrm{cm}$ of the respective TCP radiated from different sources having different values of mass (m) in $\mathrm{g}$,

$$
\begin{aligned}
& \mathrm{h}=\text { Planck's quantum constant }=6.63 \times 10^{-27} \mathrm{erg} \cdot \mathrm{sec}, \\
& V_{p r}=\text { present volume of the universe }=\left(\frac{4}{3}\right) \pi\left(\frac{c}{H_{0}}\right)^{3}=1.367 \times 10^{88} \mathrm{~cm}^{3}, \\
& H_{0}=\text { present value of Hubble's parameter }=2.023988 \times 10^{-19} \mathrm{sec}^{-1}, \\
& \mathrm{c}=\text { free-space velocity of light }=3 \times 10^{10} \mathrm{~cm} / \mathrm{sec}, \\
& c / H_{0}=\mathrm{L} \text { (Hubble length) }=\text { radius of the universe }=\mathrm{R}=1.482 \times 10^{29} \mathrm{~cm} .
\end{aligned}
$$

Here $\mathrm{m}=$ radiant mass of the universe $(=\mathrm{MR})=V_{p r} . \rho_{r}=1.16025 \times 10^{53} \mathrm{~g}$,

$\rho_{r} .=$ radiant density as per Stephan-Boltzmann formula indicated by Gamow [13] $=8.5 \times 10^{-36} T^{4} \mathrm{~g} / \mathrm{cm}^{3}$.

Pal et al [3] showed that the quantized energy $\left(\varepsilon_{T}\right)$ of TCP represents universal consciousness.

This universal consciousness exists throughout the universe in the form of universe wide web (uww) covering fields, particles, space-time continuum, dark matter, dark energy, void and all its known and unknown parameters along with all its inhabitants (with or without consciousness). This universal consciousness is to be taken into account, but usually ignored.

\section{Change in the Value of 'Fine Structure Constant' $(\alpha)$}

Are all the (measurable) dimensionless parameters that characterize the physical universe calculable in principle or are some merely determined by historical or quantum mechanical accident and incalculable?

Einstein put it more crisply: Did God have a choice in creating the universe? Imagine the Old One sitting at His control console, preparing to set off the Big Bang. "How 
fast should I set the speed of light?" "How much charge should I give to this little speck called an electron?" "What value should I give to Planck's constant, the parameter that determines the size of the quanta in which energy shall be parceled?" Do the values have to be what they are because of a deep, hidden logic?

If the square of the charge of the electron is divided by the speed of light times Planck's constant, all the dimensions (mass, time and distance) cancel out, yielding a so-called 'pure number'---alpha (fine structure constant) which is just slightly over $1 / 137$. But why it is not precisely $1 / 137$ or some other value entirely? Physicists and even mystics have tried in vain to explain why.

$$
\alpha=\mathrm{e}^{2} / \mathrm{c} \times \mathrm{h}>1 / 137
$$

Webb et al [14-16] explained that the value of $\alpha$ is higher than what it was six billion years ago. The 'fine structure constant' is now 10 parts in a million, or $0.001 \%$, bigger. According to him, 6 billion years ago the value of 'finestructure constant" was about $1 / 100000$ times less than the present value of $7.297351 \times 10^{-3}$.

This means, in effect, the operating manual of the cosmos has not been the same in all the ages. Webb and his collaborators used the powerful Keck Telescope in Hawai to look at ancient light from quasars shining through 3 to 10-billion-year-old clouds of cosmic dust. Comparing the light absorbed by atoms in the primordial clouds with that absorbed by modern atoms, they found a tiny deviation that could be explained only if the 'fine structure constant' is increasing. According to him, the value of 'c', the freespace velocity of light is not constant---it increases with the increase of the age of the universe.

The 'fine structure constant' sets the strength of the interaction between light and matter. If its value had been very different, atoms of elements would not be stable at all, and, as physicists can calculate, a small difference in the value would alter the properties of those atoms considerably.

Constants of Nature are important because it is they who determine the ultimate shape of this Universe. If the value of $G$ had been a bit different from what it is now, this Universe would have been a totally different place altogether. The constancy of $\mathrm{c}$ and its status as a universal speed limit are the foundations of the theory of relativity.

Webb et al [14-16] feels that quasar results can be explained in another way also. "Either the fundamental particles behaved differently in the past or the speed of light differed", says he. Joao Magueijo [17] of the Imperial College in London thinks changing speed of light requires rethinking the foundations and language of physics. According to them, varying speed of light could provide alternative solutions to some long-standing cosmic puzzles.

The observation by Webb et al [14-16] and his collaborators indicates that the fine structure constant---a number that determines the strength of electromagnetic force and thus the speed of light---may have been ever so slightly smaller billions of years ago. If true, the current theories are incorrect because they maintain that light's speed and other fundamental properties do not change in either space or time. New observations indicated that one of nature's supposedly immutable constants has changed over the 15 billion-year history of the Universe. Physicists were shocked at the discovery, but pleasantly so because it suggests about how the universe works on the subatomic scale may be correct.

This is actually good news for physicists, because proposed theories can accommodate changes in the fine structure constant over time. Known as string theories, they allow either a 10- or 26-dimensional universe, rather than a 4-D one containing the three spatial dimensions plus time. The extra dimensions would be curled or folded, so they would be impossible to detect in everyday life---or even in any physics experiment yet conducted.

Quasars are so far away---about 12 billion light-years--that light they produced at the dawn of the universe is only now reaching earth. During its long journey, the light has passed through clouds of intergalactic gas, where some of it has been absorbed. The patterns of absorption tell scientists something about the gas, and something about the light as well---including its speed and the fine structure constant that determines how fast it goes. The scientists hope to confirm their results using a different telescope, perhaps the Very Large Telescope at the European Southern Observatory in Chile.

The 'fine-structure constant' may have a role to play in the Pioneer problem. Quintessence, a form of universal energy which is believed to be causing the expansion of the universe may be affecting the onward journey of Pioneer 10 and 11. It is possible that whatever caused variation of the fine-structure constant in the distant past might have a present-day manifestation and thereby exerting the pull on Pioneer 10 and 11.

Y.H. Sanejouand [18, 19] expressed: Some empirical evidences in favor of the hypothesis that the speed of light decreases by a few centimeters per second each year are examined. Lunar laser ranging data are found to be consistent with this hypothesis, which also provides a straight forward explanation for the so-called Pioneer anomaly, that is, a time-dependent blue-shift observed when analyzing radio tracking data from distant spacecrafts, as well as an alternative explanation for both the apparent time-dilation of remote events and the apparent acceleration of the Universe. The main argument against this hypothesis, namely, the constancy of fine-structure and Rydberg constants, is discussed. Both of them being combinations of several physical constants, their constancy imply that, if the speed of light is indeed time-dependent, then at least two other "fundamental constants" have to vary as well. This defines strong constraints, which will have to be fulfilled by future varying-speed-of-light theories. 


\section{Discussions and Conclusion}

\subsection{Discussions}

We know that

$$
\text { Planck's constant }=\mathrm{h}=\lambda \mathrm{x} \mathrm{mv}
$$

where $\mathrm{h}=$ Planck's constant, $\lambda=$ wave-length, $\mathrm{m}=$ mass, $\mathrm{v}$ = velocity.

Planck's constant (h) is thus the product of momentum of an electron and its wave-length. Electrons retain a certain degree of freedom of motion which seems to be built into their nature. The measure of this freedom is Planck's constant. This provides the fundamental basis of particle physics. Thus, like the 'fine structure constant', if physicists explore the possibility of variation within Planck's constant, then they must be ready to come up with an alternative set of theories which would lay the foundation of a new branch of particle physics.

Motz Lloyd [20] expressed that Wheeler's entire argument rests on the existence of the constant of action $h$, from which Wheeler concludes that the constants of nature change in an unpredictable way every time the universe is reprocessed after its collapse.

What good is a universe without awareness of that universe? 'Consciousness' is to be taken into account. The quantized energy $\left(\varepsilon_{T}\right)$ of TCP represents universal consciousness that is to be taken into consideration. Thus the fundamental expression expressing the evolution of the universe from the 'void' can be written as

$$
\text { Void } \rightarrow \mathrm{E}_{\mathrm{T}}={ }_{\mathcal{E}_{T}}=\mathrm{m}_{\mathrm{T}} \mathrm{c}^{2}=\mathrm{h \nu}_{\mathrm{T}}=\mathrm{hc} / \lambda_{\mathrm{T}}=4.95 \times 10^{-16} \mathrm{erg}
$$

where,

$$
\begin{aligned}
& E_{T}=\text { total energy of the universe, } \\
& h=\text { Planck's quantum constant }=6.63 \times 10^{-27} \mathrm{erg} . \mathrm{sec}, \\
& \mathrm{v}_{\mathrm{T}}=\text { frequency of the TCP }=7.466 \times 10^{10} \mathrm{cps} .
\end{aligned}
$$

\subsection{Conclusion}

In the equation (6), the radiant mass of the universe is denoted by $\mathrm{M}_{\mathrm{R}}$. It is found from the equation (6) that $\varepsilon_{T}$ is dependent on $M_{R}$ which, in turn, depends on $V_{p r}$. $\rho_{r}$. If $V_{p r}$, the present volume of the universe increases, then $\rho_{r}$, the present radiant density would have to decrease accordingly if $\mathrm{M}_{\mathrm{R}}$ remains fixed. If $\mathrm{M}_{\mathrm{R}}$ varies, then $\mathcal{E}_{T}$ varies and thereby, $v_{\mathrm{T}}$ varies if $\mathrm{h}$ is a constant. Thus $\mathcal{E}_{T}$ is a constant only if $\mathrm{M}_{\mathrm{R}}$ is a constant. It is found from the equation (9) that if $\mathrm{h}$ varies, then $v_{\mathrm{T}}$ varies when $\boldsymbol{\varepsilon}_{T}$ is a constant at constant $\mathrm{M}_{\mathrm{R}}$. Further, if $\mathrm{h}$ is a constant, $\mathrm{v}_{\mathrm{T}}$ is also a constant when $\mathcal{E}_{T}$ is a constant at constant $\mathrm{M}_{\mathrm{R}}$. Again, $\mathcal{E}_{T}$ is directly proportional to $\mathrm{c}$, the free-space velocity of light when the proportionality constant $\mathrm{K}=\mathrm{h} / \lambda_{\mathrm{T}}$ where $\lambda_{\mathrm{T}}$ is the wavelength of TCP radiated from the radiant mass of the universe and this $\lambda_{\mathrm{T}}$ as well as $\varepsilon_{T}$ is found to be responsible for maintaining the universal temperature.

Pal et al [3] showed that the quantized energy $\left(\varepsilon_{T}\right)$ of TCP is responsible to cause the cosmic microwave background radiation temperature as well as the universal consciousness. The universal temperature is this cosmic microwave background radiation temperature that is maintained by the quantized energy $\left(\varepsilon_{T}\right)$ of TCP.

It is evident through the expression: $\varepsilon_{T} \lambda_{\mathrm{T}}=$ hc that when $\lambda_{\mathrm{T}}$ is fixed for maintaining the universal temperature, then $\mathcal{E}_{T}$ is also a universal constant. It is to be noted that the product of two constants should also be a constant. Thus the product of $c$ (natural constant of relativity) and $h$ (natural constant of quantum mechanics) should definitely be a natural constant also and thus it signifies that both the $\lambda_{\mathrm{T}}$ and $\varepsilon_{T}$ are simultaneously liable to be natural constants to maintain the universal temperature.

The universe will have to exist along with the universal consciousness as well as the cosmic microwave background radiation temperature that is maintained by the quantized energy $\left(\varepsilon_{T}\right)$ of TCP.

Further, $\varepsilon_{T}$ is found to be directly proportional to $\mathrm{c}$ where the proportionality constant, $\mathrm{K}=\mathrm{h} / \lambda_{\mathrm{T}}$. It is probable that during the gradual slowing down of temperature by the way of expansion of the universe, either the value of $\mathrm{c}$ or $\mathrm{h}$ or both the values of $\mathrm{c}$ and $\mathrm{h}$ would have to change to balance reduction in temperature of the universe. In this way the product of $\mathrm{h}$ and $\mathrm{c}$ may be slightly reduced with time for which there is a slight increase in the value of 'fine structure constant' at present.

\section{References}

[1] D. Pal and A.U. De, Physics of consciousness and its model may provide guidelines to solve Many scientific problems. Neuroquantology 1: 17-28(2004)

[2] D. Pal and A.U. De, Consciousness model: Significance of thought-carrying particles and thought-retaining particles in quantum measurement as well as cognitive problem. Neuroquantology 2: 115-116 (2005)

[3] D. Pal and A.U. De, The cosmic microwave background radiation temperature signifying the existence of the thought-carrying particle, thought retaining particle and thought force. NeuroQuantology 10: Issue3; 428-442 (September 2012)

[4] M. Bhaumik, Code Name GOD. (Penguin Books India Pvt. Ltd., 11 Community Centre, Panchsheel Park, New Delhi 110 017, India) pp. 132-133; 161-162;167; 171; 177; 183 and 184. 185-186; 89-190; 198 (2006)

[5] Dhananjay Pal, Existence of Thought Force and Its Characteristics, American Journal of Physical Chemistry. Vol. 2, No. 5, 2013, pp. 94-104. doi: 10.11648/j.ajpc.20130205.13

[6] V. F. Weisskopf, The Origin of the Universe, The World of Physics, (Simon and Schuster, 1230 Avenue of Americas, New York 10020) 3, pp. 10 and 314 (1987) 
[7] D. Pal, Existence of universal consciousness and its characteristics. Accepted for publication in 2014 International Conference on Advanced Education and Management (ICAEM2014) Beijing, China (2013)

[8] I. Prigogine and G. Nicolis, Self-Organization in NonEquilibrium Systems. (Wiley 1977) ISBN 0471024015.

[9] R. Nelson, Director, Global Consciousness Project, Princeton, New Jersey. (2009) rdnelson@princeton.edu

[10] D. Radin, The Conscious Universe: The Scientific Truth of Psychic Phenomena. (Harper Edge) (1997) ISBN 0-06251502-0.

[11] R. Penrose, Shadows of the Mind: A Search for the Missing Science of Consciousness. (Oxford University Press) (1994)

[12] W. Van De Bogart, Earth portals: Exploring New Metaphors of Consciousness 1993 willard@earthportals.com

[13] G. Gamow, The Great Expansion, The World of Physics, (Simon and Schuster, 1230 Avenue of Americas, New York 10020, New York 10020) 3:271 (1987)

[14] J.K. Webb et al. Further Evidence for Cosmological Evolution of the Fine Structure Constant. Phys. Rev. Lett, 87, 091301, (2001) (astro- ph/0012539)
[15] J.K. Webb et al. Relativistic Effects in NiII and the Search for Variation of the Fine Structure Constant. Phys. Rev. A, 63, 042509, (2001), (astro-ph/0012422121)

[16] J.K. Webb et al. Possible Evidence for a Variable Fine Structure Constant from QSO Absorption Lines: Systematic Errors. MNRAS, 327, 1223, (2001), (astro- ph/0012420)

[17] João. Magueijo, "New varying speed of light theories", Reports on the Progress of Physics, 66, 2025 (2003) (abstract: http://arxiv.org/abs/astro-ph/0305457

[18] Y.H. Sanejouand,: "About some possible empirical evidences in favor of a cosmological time variation of the speed of light", EPL vol.88, 59002 ; arXiv:0908.0249 (physics.gen-ph). (2009)

[19] Y.H. Sanejouand, "A simple varying-speed-of-light hypothesis is enough for explaining high-redshift supernovae data" arXiv:astro-ph/050958 (astro-ph) (2005)

[20] Motz Lloyd, The end of the Universe and the end of Time, The World of Physics, Simon and Schuster,1230 Avenue of Americas, New York 10020; Vol-III, P-650-652 (1987) 